\title{
Comparing the approach of a rigid sphere and a deformable droplet towards a deformable fluid surface
}

\author{
Volkert W.A. de Villeneuve*, Dirk G.A.L. Aarts ${ }^{1}$, Henk N.W. Lekkerkerker \\ Van't Hoff Laboratory, Debye Research Institute, University of Utrecht, Padualaan 8, 3584 CH Utrecht, The Netherlands \\ Received 15 November 2005; received in revised form 9 January 2006; accepted 12 January 2006 \\ Available online 10 March 2006
}

Dedicated to Professor Ivan B. Ivanov (LCPE, University of Sofia) on the occasion of his 70th birthday.

\begin{abstract}
We compare the low Reynolds number approach of rigid spheres and deformable droplets of similar sizes towards a deformable fluid interface in a fluid-fluid demixing colloid polymer system. The droplets appear during the final stages of phase separation. A small amount of rigid poly(methyl methacrylate) PMMA spheres is added to the top of the sample when phase separation has (nearly) completed. Both spheres and droplets sediment towards the interface. Far away from the surface the (dimensionless) sedimentation velocity of the droplets is slightly higher (by a factor of $\sim 1.07$ ) than that of the spheres, since the friction experienced by the droplets is slightly reduced by inner fluid circulation. Closer to the surface velocities are strongly reduced and the effective friction increases. Furthermore, the fluid surface becomes distorted at droplet/sphere to surface separations of about one diameter. The droplets strongly deform as well. Surprisingly, the velocities of rigid spheres and droplets converge. We attribute this to an increase of the friction factor of the droplet due to droplet deformation. These data may assist in a better understanding of the transport of matter through interfaces and the process of droplet coalescence. Moreover, the similarity of the drainage of rigid spheres and fluid droplets close to the surface supports the idea that from a theoretical point of view the (simpler) drainage problem for rigid spheres already captures some of the physics. (C) 2006 Published by Elsevier B.V.
\end{abstract}

PACS: 47.15.Gf; 82.70.Dd

Keywords: Droplet approach; Rigid sphere approach; Colloid-polymer mixtures

\section{Introduction}

Both the transport of matter through a fluid interface and the process of droplet coalescence are phenomena frequently observed in everyday life. They are important in a wide variety of (industrial) processes and natural phenomena, see for example [1-9]. Recent advancements in the field of microfluidics [10], in which fluids are manipulated on a microscopic level and miniature chemical reactions can be carried out on a chip, raise a further need to study these issues.

Both the passage of a sphere through an interface and the coalescence of a droplet can be considered to consist of three

\footnotetext{
* Corresponding author. Tel.: +31 30253 2391; Fax: +31 302533870 .

E-mail addresses: v.w.a.devilleneuve@ chem.uu.nl (Volkert W.A. de Villeneuve), dirk.aarts@1ps.ens.fr (Dirk G.A.L. Aarts), h.n.w.lekkerkerker@chem.uu.nl (Henk N.W. Lekkerkerker)

${ }^{1}$ Present address: Laboratoire de Physique Statistique de l'Ecole Normale Suprieure, Paris, 24, rue Lhomond - 75231 Paris Cedex 05, France.
}

stages: (i) film drainage of the continuous phase between the sphere/droplet and the surface, (ii) rupture of the film, and (iii) either the further movement of the sphere or the extrusion of the droplet material into its bulk phase. The aim of this paper is a direct comparison of step (i) of both phenomena. For droplets this first step has been studied for quite some time [11-15], but it theoretically still poses some difficulties [16]. For rigid spheres it has mainly been treated as an approximation of the first step in droplet coalescence [11,13,14,17], but it has also received some attention as the initial stage of transport through an interface [18-21]. Here, we hope that the direct comparison may shine some light on the drainage problem in droplet coalescence and at the same time is interesting at itself.

As our system we use fluorescent droplets and spheres of similar sizes and essentially made up of the same material approaching the gas liquid interface of a phase-separated colloid/polymer mixture, which makes the system rather special. Moreover, the intrinsic slowness of the colloid-polymer mixture enables a di- 
rect comparison of both processes close to the interface and ensures that the Reynolds number remains much smaller than unity. The respective approaches are imaged by laser scanning confocal microscopy (LSCM).

We will start by sketching the theoretical background in Section 2, followed by a description of the experimental method and materials in Section 3. Results are shown in Section 4, the analysis and discussion are presented in Section 5 and we conclude in Section 6.

\section{Theoretical background}

Far away from a surface, a droplet or rigid sphere with radius $R$ of $R_{\mathrm{d}}$ or $R_{\mathrm{S}}$, respectively, sediments with a constant velocity $u_{\mathrm{s}}$. The sedimentation velocity is proportional to $g \Delta \rho R^{2} / \eta$, with $g$ earth's acceleration, $\Delta \rho$ the density difference between the droplet/sphere and its surrounding phase (its buoyancy) and $\eta$ the viscosity of the phase through which the object is sedimenting. In general, for spherical objects the sedimentation velocity is given by the Rybczynski-Hadamard result, which can be found in [22],

$u_{\mathrm{s}}=\frac{2 g \Delta \rho R_{\mathrm{d}}^{2}}{3 \eta_{\mathrm{o}}} \frac{\eta_{\mathrm{o}}+\eta_{\mathrm{i}}}{2 \eta_{\mathrm{o}}+3 \eta_{\mathrm{i}}}$,

where $\eta_{\mathrm{o}}$ is the viscosity outside and $\eta_{\mathrm{i}}$ the viscosity inside the object. This equation accounts for circulation of fluid inside the object as well. When $\eta_{\mathrm{i}} \gg \eta_{\mathrm{o}}$ the well-known Stokes equation for a rigid sphere is obtained

$u_{\mathrm{s}}=\frac{2 g \Delta \rho R^{2}}{9 \eta}$,

and we recognize the Stokes friction factor as well: $f=F / u_{\mathrm{s}}=$ $6 \pi \eta R$ with $F$ the force acting on the sphere. We can estimate at what size droplets will deform by considering the capillary number $C a$, which reads

$C a=\frac{\eta u}{\gamma}$,

and we here set $u=u_{\mathrm{s}}$. Approximately, the droplet will not deform if the capillary number $\mathrm{Ca}$ remains smaller than unity and by filling in the estimate of $u_{\mathrm{s}}$ given above we obtain the Bond number $B o$ :

$B o=\frac{g \Delta \rho R^{2}}{\gamma}$.

Hence, when $B o<1$ or $R<L_{\mathrm{c}} \equiv \sqrt{\gamma / \Delta \rho g}$, droplets will not deform. We see that the cross-over length is equal to the capillary length $L_{\mathrm{c}}$. From the well-known Reynolds number,

$R e=\frac{\rho u L}{\eta}$,

with $\rho$ the mass density and $L$ the characteristic length we can estimate whether or not inertial terms become important during sedimentation. Using the estimate of the sedimentation velocity and filling in typical numbers of $\rho \sim 10^{3} \mathrm{~kg} \mathrm{~m}^{-3}$, $\Delta \rho \sim 10^{2} \mathrm{~kg} \mathrm{~m}^{-3}, L \equiv R \sim 10^{-5} \mathrm{~m}$ and $\eta \sim 10^{-2} \mathrm{~Pa}$ s we find that $R e \ll 1$ during sedimentation.
Closer to the surface the velocity of the sphere/droplet reduces. The friction factor can then be written as $f=6 \pi \eta_{\mathrm{o}} R \lambda$ with $\lambda$ the correction to the Stokes friction. For a rigid sphere approaching a solid or free non-deformable surface exact treatments are given in [23], which describe both the undistorted fall of Eq. (2) and the velocity close to the surface:

$\lambda=4 / 3 \sinh \alpha \sum_{n=1}^{\infty} \frac{n(n+1)}{(2 n-1)(2 n+3)} C(n, \alpha)$

with

$C_{\text {rigid }}(n, \alpha)=\frac{2 \sinh ((2 n+1) \alpha)+(2 n+1) \sinh (2 \alpha)}{4 \sinh ^{2}((n+1 / 2) \alpha)-(2 n+1)^{2} \sinh ^{2}(\alpha)}-1$

for a rigid surface and

$C_{\text {free }}(n, \alpha)=\frac{4 \cosh ^{2}((n+1 / 2) \alpha)+(2 n+1)^{2} \sinh ^{2} \alpha}{2 \sinh ((2 n+1) \alpha)-(2 n+1) \sinh (2 \alpha)}-1$

for a free surface, with $\alpha=\cosh ^{-1}(d / R)$ and $d$ the distance of the sphere's center to the interface. In the limit of $h \rightarrow \infty$, the friction factor $\lambda \rightarrow 1$ in both cases. In the asymptotic limit of $h=d-R \longrightarrow 0$, where $h$ is the minimal distance between sphere and surface, it becomes:

$\lambda \rightarrow \frac{R}{h} \quad$ and $\quad \lambda \rightarrow \frac{R}{4 h}$

for a solid surface and for a planar free surface, respectively. These limiting equations can be found from lubrication equations as well [24,25]. The factor 4 difference between a solid and a fluid interface is commonly observed in these type of problems. For a solid sphere approaching a deformable surface Hartland has derived expressions from lubrication theory valid for small sphere-surface separations [13]. He finds that $h \propto t^{-1 / 2}$. These results have later been confirmed by Jones and Wilson [16].

However, when both the fluid interface and the droplet get distorted, the description becomes more complicated: there is some constriction in the film thickness at its periphery, which slows down drainage. Furthermore, fluid circulates in the drop since it has a finite viscosity, which tends to speed up drainage as compared to a rigid sphere. These last two effects are properly treated by Jones and Wilson [16], who point out that these effects are not captured in simple lubrication theories. They predict several asymptotic regimes, which have been confirmed by Yantsios and Davis [26] using extended lubrication theories. No full analytical treatment can be obtained and the main problem in comparison with the present experimental data is that the asymptotic limits become valid for very small separations [26]. Furthermore, to pinpoint our problem we will only consider rigid spheres with $B o<1$, where $\Delta \rho$ now is the density difference between solid and gas, and interface deformation thus does not lead to a tailing configuration [19]. The Bond number may be viewed as the ratio between Eq. (2) and the capillary velocity. The latter is the characteristic interface velocity, which is given by the balance between the Laplace pressure and the viscous dissipation, resulting in the capillary velocity $u_{\mathrm{c}}=\gamma / \eta$. Note 

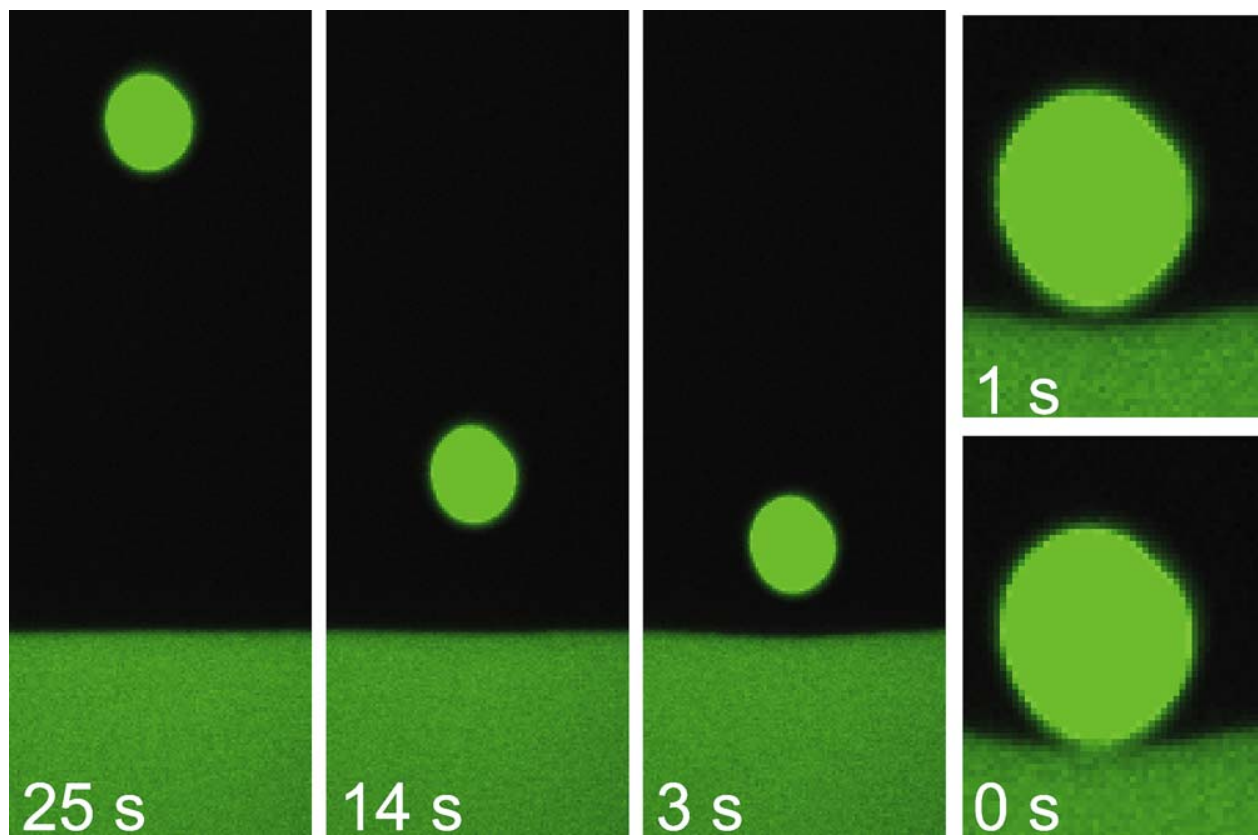

Fig. 1. Approach of a $15 \mu \mathrm{m}$ diameter sphere to the interface. Times are before wetting by the liquid phase. Initially the interface is undeformed ( $25 \mathrm{~s})$. At separations smaller than the sphere diameter the interface starts to deform and the sphere slows down $(14 \mathrm{~s})$. As the sphere approaches the interface, the separation becomes more pronounced ( 3 and $2 \mathrm{~s})$. At very small separations the liquid phase wets the sphere and pulls down the sphere( 1 and $0 \mathrm{~s})$. The analogy between sphere and droplet stops here.

that the capillary velocity depends on the viscosity of both the gas and the liquid phase, whereas the bulk sedimentation rate of rigid spheres only depends on the viscosity of one phase (here, it is the gas). The viscosity of the phases therefore does somewhat affect the Bond number, and therefore the extent of surface deformation.

Finally, it can easily be shown that during the deformation of the interface and droplet the Reynolds number remains much smaller than unity. Since the Bond number is smaller than unity in all cases, the largest velocity of importance is the capillary velocity. Setting $u$ in Eq. (5) equal to $u_{\mathrm{c}}$ we can estimate at what length inertia becomes as important as viscous dissipation. The length $L_{\eta}$ at which the inertial contribution starts to dominate over viscous terms therefore is:

$L_{\eta}=\frac{\eta^{2}}{\rho \gamma}$.

Compared to a 'normal' fluid, a substantial increase in viscosity or decrease in interfacial tension is necessary to access the viscous regime. Here, the interfacial tension is decreased by resorting to colloid-polymer mixtures. Owing to a depletioninduced attraction [27,28], these mixtures exhibit a "gas" (colloid poor/polymer rich)-"liquid" (colloid rich/polymer poor) phase separation when the polymer/colloid diameter ratio $q$ is
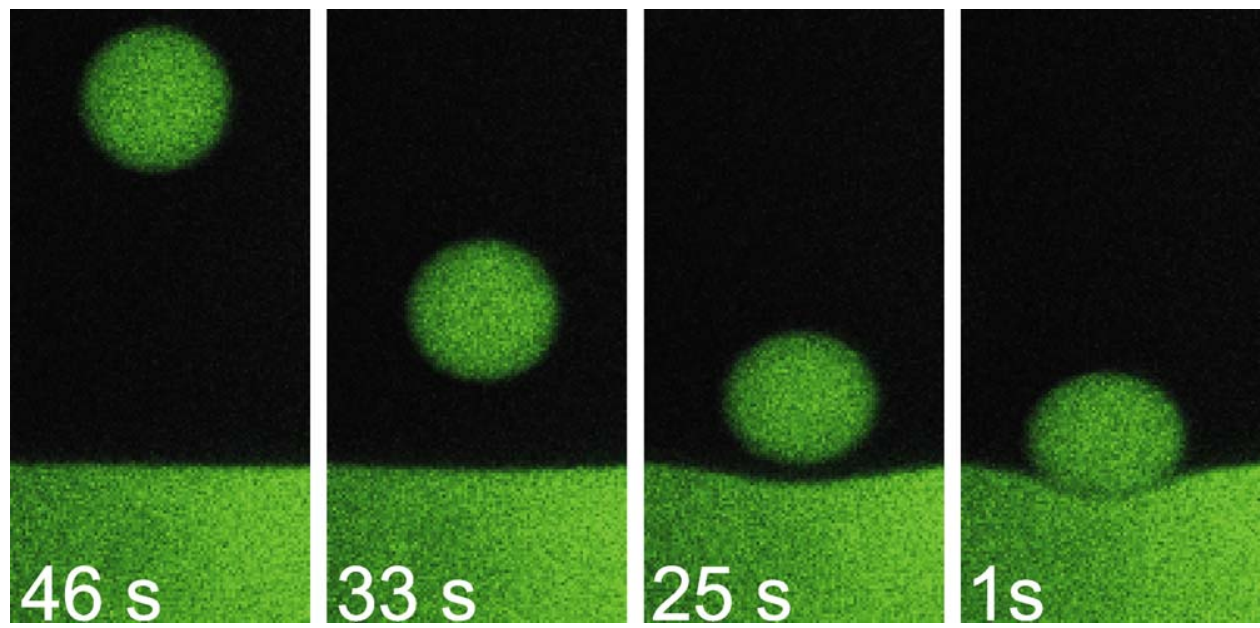

Fig. 2. Approach of a $33 \mu \mathrm{m}$ diameter droplet to the interface. Times are before coalescence. Initially ( $46 \mathrm{~s})$ the interface is undeformed. At separations of approximately the droplet diameter the interface and droplet starts to deform and the droplet slows down ( $33 \mathrm{~s})$. As the droplet approaches the interface, the separation becomes more pronounced $(25 \mathrm{~s})$. Right before coalescence $(1 \mathrm{~s})$, the droplet is nearly immobile and only a very thin film separates it from its bulk phase. 
sufficiently large $\left(q=\sigma_{\mathrm{p}} / \sigma_{\mathrm{c}} \geq 0.30\right.$ [29]). Since colloidal particles have sizes of typically $1-1000 \mathrm{~nm}$, these systems have much lower interfacial tensions-as experimentally verified [30-33] - than molecular systems with a magnitude proportional to $[34,35]$ :

$\gamma \propto \frac{k_{\mathrm{B}} T}{d^{2}}$,

with $k_{\mathrm{B}} T$ the thermal energy and $d$ the typical length scale at the interface, similar to the colloidal particle diameter $\sigma_{\mathrm{c}}$ far away from the critical point. Combining Eq. (11) with Eq. (10) shows that $L_{\eta}$ can easily become of the order of meters and the viscous regime therefore is readily available for experimental inspection.

\section{Experimental system and technique}

We used a colloidal particle set of polymethyl-metacrylate (PMMA), fluorescently labeled with 4-methylaminoethyl metacrylate-7-nitrobenzo-2-oxa-1,3-diazol (NBD). The particles were prepared using the Bosma method [36], slightly modified by using decalin (Merck, for synthesis) as the reaction solvent [37]. The set's polydispersity is below $10 \%$ from electron microscopy, and the dynamic light scattering particle radius is $25 \mathrm{~nm}$. Polystyrene $\left(233 \mathrm{~kg} \mathrm{~mol}^{-1}\right)$ was added as depletant polymer, with an estimated radius of gyration $R_{\mathrm{g}}$ of $14 \mathrm{~nm}$ [38]. Very large, very polydisperse PMMA-NBD spheres $\left(\sigma_{\mathrm{c}}=100 \mathrm{~nm}-100 \mu \mathrm{m}, \quad \rho=1.17 \mathrm{~g} \mathrm{ml}^{-1}\right)$ were accidentally obtained from a synthesis following [36], and subsequently size-fractionated twice in hexane $[39,40]$. The resulting 5$40 \mu \mathrm{m}$ spheres were transferred to cis/trans decalin and diluted to a concentration of $1 \mathrm{wt} . \%$.

At a colloid volume fraction $\phi_{\mathrm{c}}=(\pi / 6) \sigma_{\mathrm{c}}^{3} n_{\mathrm{c}}=0.076$ of the $25 \mathrm{~nm}$ colloids and a polymer volume fraction $\phi_{\mathrm{p}}=$ $4 / 3 \pi R_{\mathrm{g}}^{3} n_{\mathrm{p}}=0.50$ the system phase separated into a colloidrich (colloidal liquid) and a polymer rich (colloidal gas) phase (with $n_{\mathrm{c}}$ and $n_{\mathrm{p}}$ the number of colloids and polymers, respectively) [41]. The densities of the phases were 0.90 and $0.95 \mathrm{~g} / \mathrm{ml}$ with viscosities of 8 and $31 \mathrm{mPa}$ for the gas and liquid phases, respectively. By analyzing the interfacial profile close to a hard wall the capillary length can be obtained $[33,42]$. Since the densities of both phases are known, the surface tension can be estimated from the capillary length: $0.16 \mu \mathrm{N} \mathrm{m}^{-1}$. By analyzing the capillary wave spectrum we found $\gamma=0.2 \mu \mathrm{N} \mathrm{m}^{-1}$ [43] in good agreement with the more suitable interfacial profile technique.

A Nikon Eclipse E400 laser scanning confocal microscope was placed horizontally to study the colloid polymer mixture [37]. The setup allows for monitoring using transmission light microscopy as well as laser scanning confocal microscopy (LSCM). A confocal scanning laser head (Nikon C1) was mounted on the microscope with a maximal capturing rate of approximately one frame $(512 \times 512$ pixels $)$ per second. The microscope was furthermore equipped with a $488 \mathrm{~nm}$ ArKr laser and several lenses (NIKON 20× ELWD Plan Fluor (NA 0.45) and $60 \times$ CFI Plan Apochromat (NA 1.4)). The sample container is a small glass vial, part of which is removed and replaced by a thin $(0.17 \mathrm{~mm})$ glass wall.
The colloid-polymer mixture was left until a sharp interface had formed, after approximately $20 \mathrm{~min}$ [41]. Drops of colloidal liquid subsequently formed at the colloidal gas-air interface, detached from it by gravity and subsequently sedimented towards the gas-liquid interface until they coalesced with it [44].

A small amount (1.0\% v/v of the total sample volume) of very large, very polydisperse PMMA-NBD was carefully added to the top of colloidal gas phase, $16 \mathrm{~h}$ after phase separation had completed. The spheres subsequently sedimented towards the gas-liquid interface.

\section{Observations}

Examples of spheres and droplets approaching the interface are shown in Figs. 1 and 2. Both types of objects initially sediment at a constant velocity. They start to slow down due to solvent backflow at a separation of about their own diameter and the gas-liquid interface and the droplet surface then get distorted as well. The velocity continues to decrease strongly and the surface distortion becomes more pronounced. The spheres then either break through the interface and enter a tailing mode

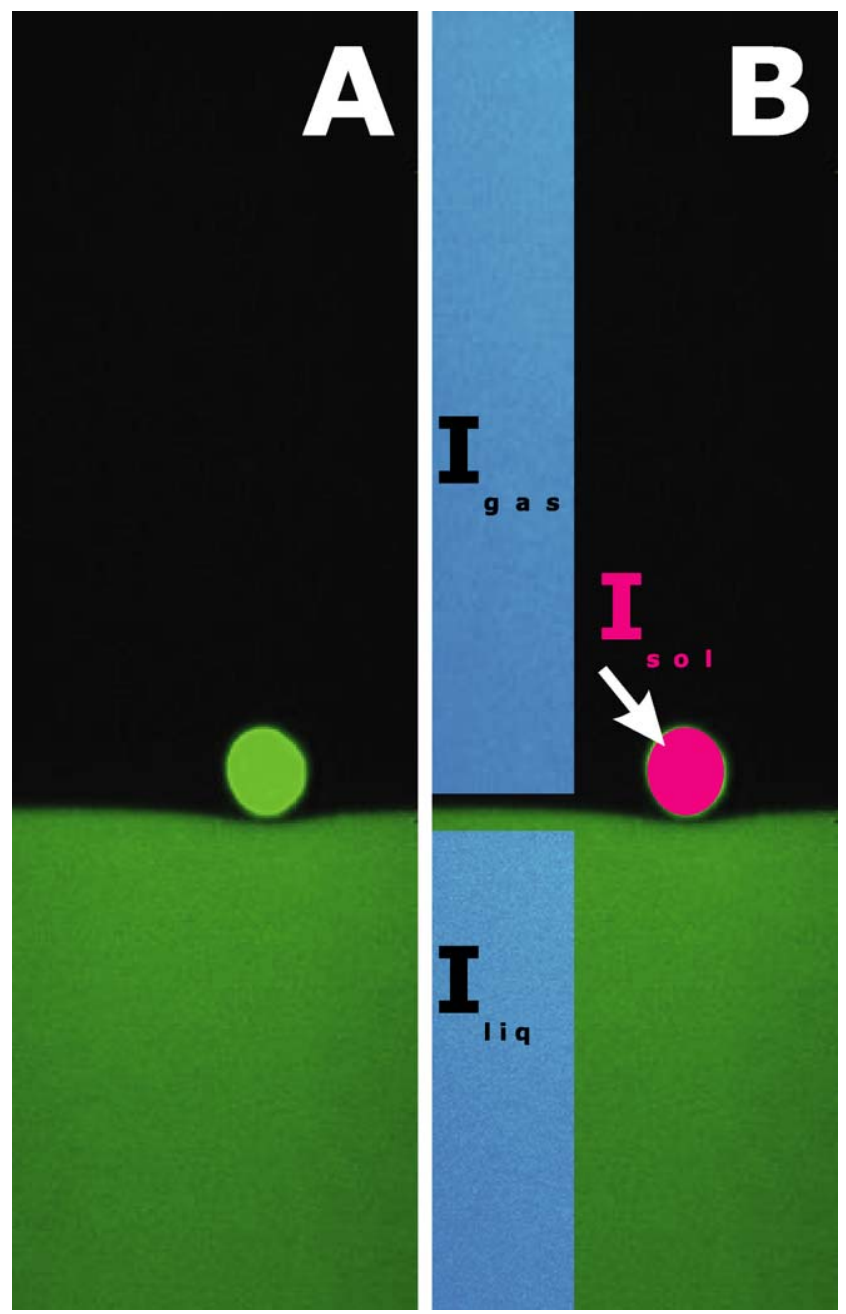

Fig. 3. (A) Sample image of a sphere approaching the gas-liquid interface. (B) the gas/liquid cutoff intensity is based on the average intensity of the bulk phases. The solid phase is assigned to pixels with intensities higher than the maximum intensity found in the liquid phase. 
or become wetted by the liquid phase and are dragged into it. The critical sphere radius for which $B o=1$ is $\sim 8 \mu \mathrm{m}$, but we observe that even slightly larger spheres do not enter a tailing configuration [19]. The wetting occurs at about sphere-surface separations below $1 \mu \mathrm{m}$ and the sphere is pulled down due to the interfacial tension, see Fig. 1 ( $1 \mathrm{~s}$ and $0 \mathrm{~s}$ ). Both the tailing mode and the wetting of the sphere lie outside the scope of the present paper and will be discussed elsewhere. Droplets will eventually coalesce with the bulk liquid phase. There, the thin draining gas-film breaks, which is facilitated by the dynamic interface roughness [43]. The subsequent suction of the droplet into the bulk liquid is purely a capillary-viscous affair [45].

In the next section we will quantitatively analyze and discuss these observations.

\section{Analysis and discussion}

To investigate droplet and surface deformation and droplet and sphere movement, IDL routines [46] were developed to as-
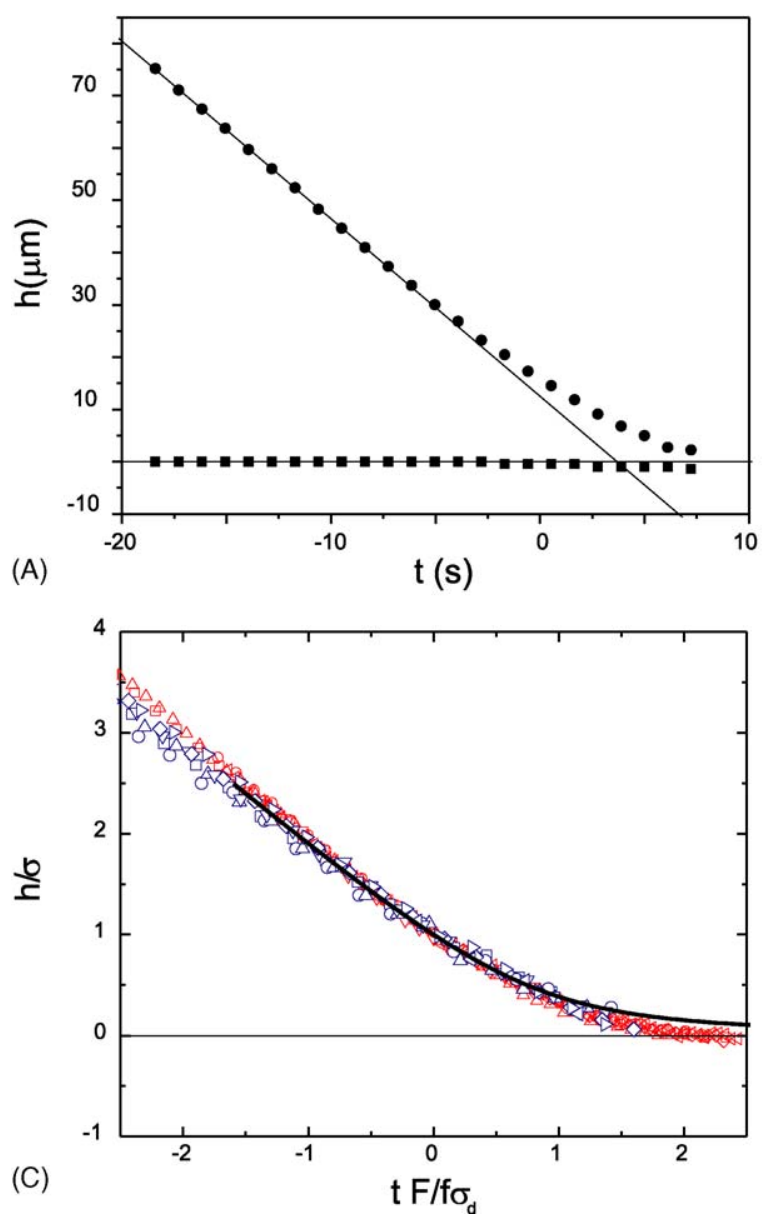

sign gas, liquid and solid phases to each of the images' pixels, based on their intensity. The average intensities $I_{\text {gas }}$ and $I_{\text {liq }}$ of the bulk gas and liquid phases were used to obtain a cutoff intensity $I_{\text {cut }}=1 / 2\left(I_{\text {gas }}+I_{\text {liq }}\right)$ to distinguish between the gas and liquid phases (Fig. 3A). Pixels with intensities greater than the maximum intensity of the bulk liquid phase were assigned to the solid phase of the rigid sphere.

The (mean) radius of a sphere was obtained from its sedimentation velocity far from the interface in the gas or liquid phase (Eq. (2)). The obtained sphere velocities agree very well with the sphere radii obtained from image analysis but were sometimes slightly off because the center of the sphere was slightly out of focus.

In Fig. 4A and B the approach of a $15 \mu \mathrm{m}$ diameter sphere and a $26 \mu \mathrm{m}$ droplet are shown, respectively. The bulk sedimentation velocities obtained from Eqs. (2) and (1) agree well with the obtained values for droplets and spheres (droplets : $4.3 \mu \mathrm{m} \mathrm{s}^{-1}$ (experiment) and $4.2 \mu \mathrm{m} \mathrm{s}^{-1}$ (theory); spheres : $3.4 \mu \mathrm{m} \mathrm{s}^{-1}$ (experiment) and $3.6 \mu \mathrm{m} \mathrm{s}^{-1}$ (theory)) Once the droplets and spheres
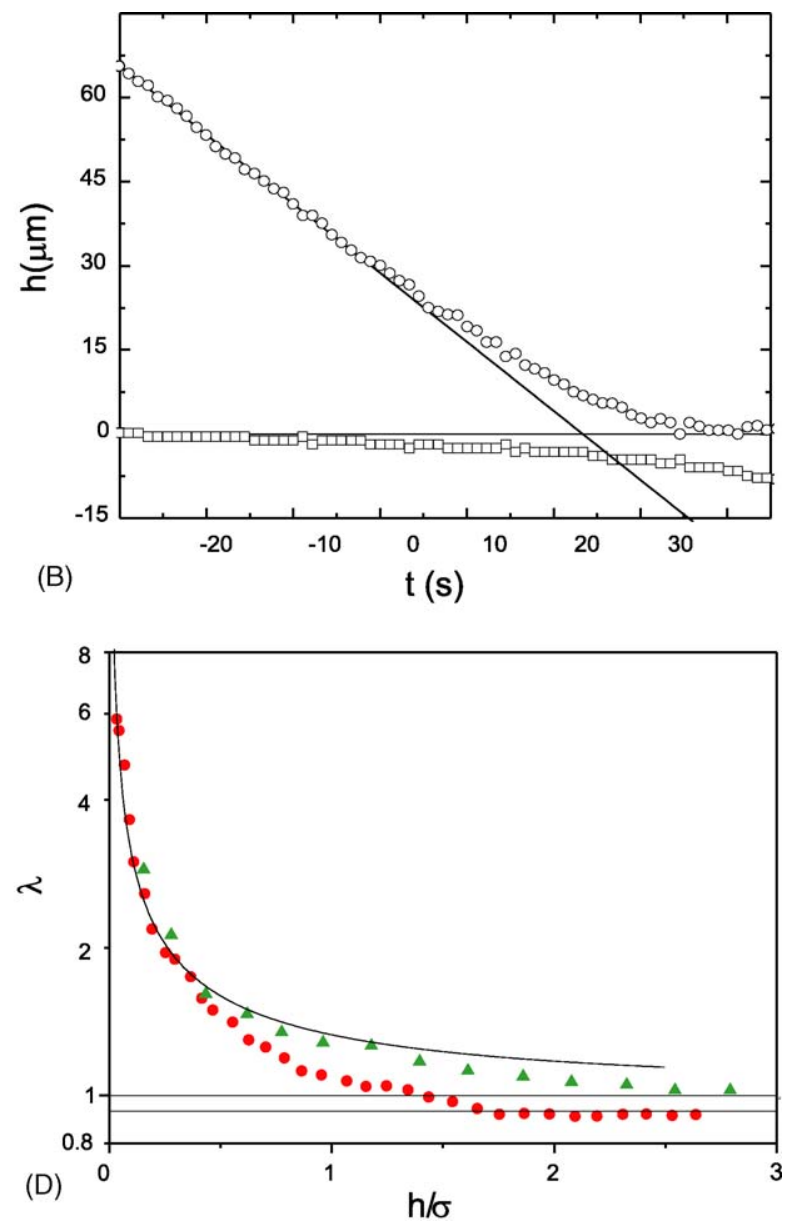

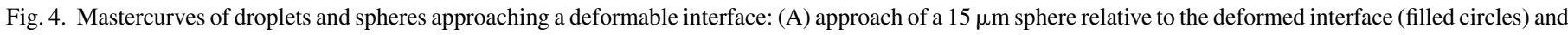

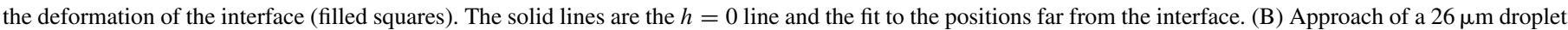

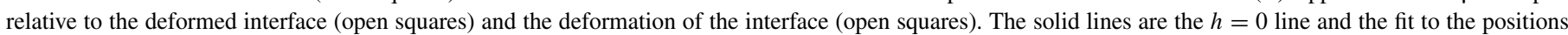

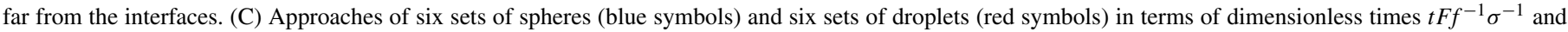

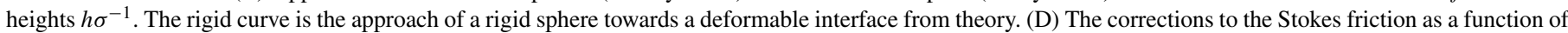

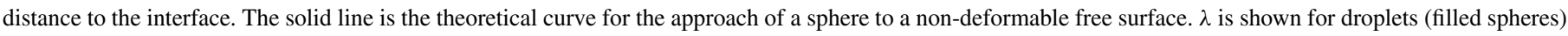

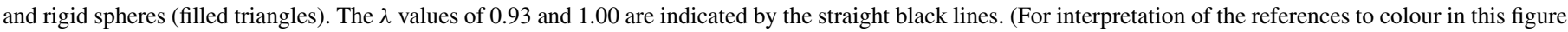
legend, the reader is referred to the web version of this article). 
get as close to the interface as a single particle diameter they slow down. Both curves, plotted in dimensionless units $(h / \sigma$ versus $F / f$ ), are quite similar to the theoretical curve of the approach of a rigid sphere towards a free surface (Fig. 4C). The larger deviations in the sphere velocity data far from the interface can be attributed to the slight deviation from the shape of a perfect sphere Fig. 4C.

The correction to the Stokes friction factor $\lambda$ for droplets and spheres is plotted as a function of distance to the interface in Fig. 4D. It is obtained by adjacent averaging and subsequent differentiation of the datapoints. The theoretical curve for the approach of a rigid sphere to a free non-deformable surface is shown for comparison (Eq. (6) and Eq. (8)). The spheres follow Eq. (6) nearly perfectly (until the spheres are wetted by the liquid phase). The minimal deformation of the interface and the incorporation of this deformation into the interface-sphere separation explain the agreement of experimental data with the theoretical curve, which does not take interface deformation into account.

The droplets approach the interface slightly faster initially, with $\lambda \sim 0.93$, in agreement with Eq. (1). As the droplet and sphere approach the interface, $\lambda$ increases due to solvent backflow, interface deformation and droplet deformation.

Interestingly, the $\lambda$ values converge at very small separations, which supports the idea that from a theoretical point of view the (simpler) drainage problem for rigid spheres already captures some of the physics of droplet coalescence. The similarity is striking since the approach of a rigid sphere towards a rigid surface and a free surface result in very different friction factors (Eq. (9)). Apparently, a second free surface (i.e. the droplet's surface) instead of a rigid surface does not affect the friction factor as strongly. We attribute the convergence of the velocities to the deformation of the droplet, which gradually cancels out the higher droplet velocity, caused by fluid circulation.

\section{Conclusion}

We have experimentally studied the approach of similarly sized droplets and rigid spheres to a colloidal gas-liquid interface. A special feature of our experimental system is that the droplets and spheres are essentially made up of the same material. As expected, the droplet approaches the interface slightly faster than the rigid sphere. Interestingly, close to the interface the approach velocities converge. This supports the idea that from a theoretical point of view the (simpler) drainage problem for rigid spheres already captures some of the physics.

\section{Acknowledgements}

This work is dedicated to Ivan Ivanov and his inspiring contributions to the physics and hydrodynamics of interfaces. Roel Dullens, Hans Scherff and Esther Groeneveld are acknowledged for particle synthesis. This work was supported by the Stichting voor Fundamenteel Onderzoek der Materie (Foundation for Fundamental Research on Matter), which is part of the Nederlandse Organisatie voor Wetenschappelijk Onderzoek (Netherlands Organization for Advancement of Research). Support of VWAdV by the DFG through the SFB TR6 is acknowledged.

\section{References}

[1] L.F. Phillips, Acc. Chem. Res. 37 (2004) 982.

[2] E. Dickinson, J. Dairy Sci. 80 (10) (1997) 2607-2619.

[3] K.L. Hoy, J. Coat. Technol. 68 (853) (1996) 33-39.

[4] W.T. Shin, S. Yiacoumi, C. Tsouris, Curr. Opin. Colloid Interf. Sci. 9 (3-4) (2004) 249-255.

[5] L.J. Lewis, P. Jensen, J.L. Barrat, Phys. Rev. B 56 (4) (1997) 2248 2257.

[6] A.E. Brandes, G.F. Zhang, J. Vivekanadan, J. Appl. Meteorol. 43 (2004) 461.

[7] H.J. Stechemesser, A.V. Nguyen, Int. J. Miner. Process. 56 (1999) $117-132$.

[8] R. Lande, A.M. Wood, Deep-Sea Res. 34 (1987) 61-72.

[9] M. Manga, H.A. Stone, R.J. O'Connell, J. Geophys. Res. 98 (1993) 19979 19990.

[10] H.A. Stone, A.D. Stroock, A. Ajdari, Annu. Rev. Fluid Mech. 36 (2004) 381.

[11] S. Hartland, J. Colloid Interf. Sci. 26 (1968) 383

[12] S. Hartland, Can. J. Chem. Eng. 47 (1969) 221.

[13] S. Hartland, Chem. Eng. Sci. 24 (1969) 987.

[14] S. Hartland, Chem. Eng. Sci. 24 (1969) 611.

[15] S. Hartland, B. Yang, S.A.K. Jeelani, Chem. Eng. Sci. 49 (1993) 1313.

[16] A.F. Jones, S.D.R. Wilson, J. Fluid Mech. 87 (1978) 263

[17] S.T. Shah, D.T. Wasan, R.C. Kintner, Chem. Eng. Sci. 27 (1972) 881-893.

[18] H.C. Maru, D.T. Wasan, R.C. Kintner, Chem. Eng. Sci. 26 (1971) $1615-1628$

[19] A.S. Geller, S.H. Lee, L.G. Leal, J. Fluid Mech. 169 (1986) 27-69.

[20] O. Pitois, P. Moucheront, C. Weill, C.R. Acad. Sci., Ser. IIb: Mec., Phys., Chim., Astron. 327 (6) (1999) 605-611.

[21] O. Pitois, X. Chateau, Langmuir 18 (25) (2002) 9751-9756.

[22] H. Lamb, Hydrodynamics, Cambridge University Press, Cambridge, 1932.

[23] J. Happel, H. Brenner, Low Reynolds Number Hydrodynamics, Martinus Nijhoff Publishers, Dordrecht, 1986.

[24] O. Reynolds, Philos, Trans. R. Soc. Lond. 177 (1886) 157.

[25] G.E. Charles, S.G. Mason, J. Colloid Sci. 15 (1960) 236.

[26] S.G. Yiantsios, R.H. Davis, J. Fluid Mech. 217 (1990) 547

[27] S. Asakura, F. Oosawa, J. Chem. Phys. 22 (1954) 1255.

[28] A. Vrij, Pure Appl. Chem. 48 (1976) 471.

[29] H.N.W. Lekkerkerker, W.C.K. Poon, P.N. Pusey, A. Stroobants, P.B. Warren, Europhys. Lett. 20 (6) (1992) 559.

[30] G.A. Vliegenthart, H.N.W. Lekkerkerker, Prog. Colloid Polym. Sci. 105 (1997) 27.

[31] E.H.A. de Hoog, H.N.W. Lekkerkerker, J. Phys. Chem. B 103 (25) (1999) 5274.

[32] B.H. Chen, B. Payandeh, M. Robert, Phys. Rev. E 62 (2) (2000) 2369.

[33] D.G.A.L. Aarts, J.H. van der Wiel, H.N.W. Lekkerkerker, J. Phys.: Condens. Matter 15 (1) (2003) 245

[34] P.G. De Gennes, Scaling Concepts in Polymer Physics, Cornell University Press, Ithaca, 1979

[35] J.S. Rowlinson, B. Widom, Molecular Theory of Capillarity, Clarendon Press, Oxford, 1982.

[36] G. Bosma, C. Pathmamanoharan, E.H.A. de Hoog, W.K. Kegel, A. van Blaaderen, H.N.W. Lekkerkerker, J. Colloid Interf. Sci. 245 (2) (2002) 292.

[37] D.G.A.L. Aarts, H.N.W. Lekkerkerker, J. Phys.: Condens. Matter 16 (2004) S4231.

[38] B. Vincent, Colloids Surf. 50 (1990) 241.

[39] V.W.A. de Villeneuve, R.P.A. Dullens, D.G.A.L. Aarts, E. Groeneveld, J.H. Scherff, W.K. Kegel, H.N.W. Lekkerkerker, Science 309 (2005) 1231.

[40] V.W.A. de Villeneuve, D. Verboekend, R.P.A. Dullens, D.G.A.L. Aarts, W.K. Kegel, H.N.W. Lekkerkerker, J. Phys.: Condens. Matter 17 (2005) S3371. 
[41] D.G.A.L. Aarts, R.P.A. Dullens, H.N.W. Lekkerkerker, New J. Phys. 7 (2005) 40.

[42] D.G.A.L. Aarts, J. Phys. Chem. B 109 (2005) 7407.

[43] D.G.A.L. Aarts, M. Schmidt, H.N.W. Lekkerkerker, Science 304 (2004) 847.
[44] D.G.A.L. Aarts. The interface in demixed colloid-polymer systems: Wetting, waves and droplets. PhD thesis, University of Utrecht, 2005.

[45] D.G.A.L. Aarts, H.N.W. Lekkerkerker, H. Guo, G.H. Wegdam, D. Bonn, Phys. Rev. Lett. (2005).

[46] Interactive data language, Research Systems Inc. (http://www.rsinc.com). 